\title{
Efficacy and Safety of Pembrolizumab in Patients with Refractory Advanced Biliary Tract Cancer: Tumor Proportion Score as a Potential Biomarker for Response
}

\author{
Junho Kang, MD ${ }^{1}$ \\ Jae Ho Jeong, MD ${ }^{1}$ \\ Hee-Sang Hwang, MD2 \\ Sang Soo Lee, MD, PhD ${ }^{3}$ \\ Do Hyun Park, MD, PhD 3 \\ Dong Wook Oh, MD \\ Tae Jun Song, MD, PhD ${ }^{3}$ \\ Ki-Hun Kim, MD, PhD ${ }^{4}$ \\ Shin Hwang, MD, PhD ${ }^{4}$ \\ Dae Wook Hwang, MD, PhD 4 \\ Song Cheol Kim, MD, PhD ${ }^{4}$ \\ Jin-hong Park, MD, PhD ${ }^{5}$ \\ Seung-Mo Hong, MD, PhD² \\ Kyu-pyo Kim, MD, PhD' \\ Baek-Yeol Ryoo, MD, $\mathrm{PhD}^{1}$ \\ Changhoon Yoo, MD, PhD ${ }^{1}$
}

Departments of ${ }^{1}$ Oncology, ${ }^{2}$ Pathology,

${ }^{3}$ Gastroenterology, ${ }^{4}$ Surgery, and ${ }^{5}$ Radiation

Oncology, Asan Medical Center, University of

Ulsan College of Medicine, Seoul, Korea

Correspondence: Changhoon Yoo, MD, PhD

Department of Oncology, Asan Medical Center, University of Ulsan College of Medicine,

88 Olympic-ro 43-gil, Songpa-gu,

Seoul 05505, Korea

Tel: 82-2-3010-1727

Fax: 82-2-3010-6961

E-mail:yooc@amc.seoul.kr

Co-correspondence: Baek-Yeol Ryoo, MD, PhD Department of Oncology, Asan Medical Center, University of Ulsan College of Medicine,

88 Olympic-ro 43-gil, Songpa-gu,

Seoul 05505, Korea

Tel: 82-2-3010-3211

Fax: 82-2-3010-6961

E-mail: ryooby@amc.seoul.kr

Received August 24, 2019

Accepted December 17, 2019

Published Online December 18, 2019

*Junho Kang, Jae Ho Jeong, and Hee-Sang

Hwang contributed equally to this work.

\section{Purpose}

The current standard chemotherapy for advanced biliary tract cancer (BTC) has limited benefit, and novel therapies need to be investigated.

\section{Materials and Methods}

In this prospective cohort study, programmed death ligand-1 (PD-L1)-positive BTC patients who progressed on first-line gemcitabine plus cisplatin were enrolled. Pembrolizumab 200 mg was administered intravenously every 3 weeks.

Results

Between May 2018 and February 2019, 40 patients were enrolled. Pembrolizumab was given as second-line (47.5\%) or $\geq$ third-line therapy (52.5\%). The objective response rate was $10 \%$ and $12.5 \%$ by Response Evaluation Criteria in Solid Tumor (RECIST) v1.1 and immune-modified RECIST (imRECIST) and median duration of response was 6.3 months. Among patients with progressive disease as best response, one patient (1/20,5.0\%) achieved complete response subsequently. The median progression-free survival (PFS) and overall survival (OS) were 1.5 months (95\% confidence interval [Cl], 0.0 to 3.0 ) and 4.3 months ( $95 \% \mathrm{Cl}, 3.5$ to 5.1), respectively, and objective response per imRECIST was significantly associated with PFS $(p<0.001)$ and OS ( $p=0.001)$. Tumor proportion score $\geq 50 \%$ was significantly associated with higher response rates including the response after pseudoprogression (vs. < 50\%; $37.5 \%$ vs. $6.5 \%$; $p=0.049$ ).

\section{Conclusion}

Pembrolizumab showed modest anti-tumor activity in heavily pretreated PD-L1-positive BTC patients. In patients who showed objective response, durable response could be achieved. 


\section{Introduction}

Biliary tract cancer (BTC) is a heterogeneous group of diseases, which consists of intrahepatic cholangiocarcinoma (iCCA), extrahepatic cholangiocarcinoma (eCCA), and gallbladder cancer (GBCA) [1]. The incidence of BTC is rare in the United States and Europe, but the prevalence is higher in Asia and Latin America [2,3]. Complete surgical resection, which is the only curative treatment, is available for only a minority of patients and is hindered by a low overall 5 -year survival rate and high rate of tumor recurrence [1,4]. For patients with advanced BTC, the phase III ABC-02 and ABC06 trials showed the clinical efficacy of gemcitabine plus cisplatin (GemCis) and oxaliplatin plus fluoropyrimidine (mFOLFOX) as first-line therapy and second-line therapy, respectively $[5,6]$. However, the survival outcomes remain dismal with a median overall survival (OS) of $<1$ year, and none of the targeted agents have been approved for treatment of BTC [7-9].

Pembrolizumab is an anti-programmed death 1 (PD-1) antibody, which has shown anti-tumor activity in various types of cancers, including non-small-cell lung cancer, melanoma, gastric cancer, and urothelial cancer [10-13]. Tumor PD-L1 expression emerged as a potential biomarker of response to pembrolizumab in several types of tumors [13-15], and cancer patients with mismatch repair (MMR) deficiency are sensitive to immune checkpoint blockade, regardless of tumor origin $[16,17]$. In patients with BTC, tumor PD-L1 expression and MMR deficiency have been reported, indicating that pembrolizumab may be potentially effective in the treatment of BTC [18-21].

Although pembrolizumab has shown modest efficacy in patients with advanced PD-L1-positive BTC in prior singlearm phase I/II KEYNOTE-028 and -158 studies [22], more data are needed to evaluate the clinical outcomes of pembrolizumab in unresectable or metastatic BTC, considering the heterogeneity of the disease. In this prospective cohort study, we analyzed the efficacy and safety of pembrolizumab in patients with advanced BTC after progression on first-line GemCis.

\section{Materials and Methods}

\section{Patients}

This study is a single-center, prospective cohort study aimed to evaluate the efficacy, safety, and biomarker of pembrolizumab in patients with advanced BTC including iCCA,
eCCA, and GBCA (ClinicalTrials.gov identifier, NCT03695952). Patients with histologically or cytologically confirmed unresectable or metastatic BTC who radiologically progressed after receiving first-line GemCis were eligible for enrolment if they had PD-L1-positive tumors (PD-L1 $\geq 1 \%$ of tumor cells graded by local pathologists), aged $\geq 19$ years, and provided a written informed consent for the collection of data on baseline characteristics and clinical outcomes. Biomarker analysis using blood and tumor tissues was also required for the enrolment.

\section{Histopathological analysis}

All histologic data including PD-L1 and MMR status were centrally reviewed after enrolment to this study by an academic pathologist. The administration of pembrolizumab was not affected by the results of this central review of PD-L1 and MMR status. PD-L1 expression was assessed by conducting an immunohistochemistry of archived tumor tissues with PD-L1 immunohistochemistry SP263 (Ventana Benchmark Ultra, Tuscon, AZ) or 22C3 pharmDx kit (Agilent Technologies, Santa Clara, CA). The tumor cells were considered PDL1-positive if the viable tumor cells exhibited any perceptable, partial or complete, linear cell membrane staining. The immune cells were considered PD-L1-positive if the cells displayed any membranous or cytoplasmic PD-L1 staining [23]. Tumor proportion score (TPS) was defined as the percentage of viable tumor cells that showed partial or complete membrane staining of PD-L1 relative to all viable tumor cells present in the sample [24]. Combined positive score (CPS) was defined as the number of PD-L1-positive cells (tumor cells, lymphocytes, and macrophages) divided by the total number of viable tumor cells and multiplied by 100 [25]. Antibodies specific for MMR proteins included MLH1 (1:10, clone G16815, BD Pharmingen, San Jose, CA), MSH2 (dilution 1:100, clone FE11, Calbiochem, San Diego, CA), MSH6 (1:100, clone EP49, Novus Biologicals, Centennial, CO), and PMS2 (1:50, clone A16-4, BD Pharmingen).

\section{Treatment and assessment}

All patients received $200 \mathrm{mg}$ pembrolizumab intravenously every 3 weeks, on day 1 of each 3-week cycle, until disease progression, occurrence of unacceptable toxicity, withdrawal of consent, or physician's decision to stop treatment. The tumor response was assessed every 6 weeks and prospectively graded according to Response Evaluation Criteria in Solid Tumors (RECIST) v1.1 and immune-modified RECIST (imRECIST). At the discretion of attending physicians, continuation of pembrolizumab beyond progressive disease (PD) was allowed if there was potential clinical benefit. Pembrolizumab dose reductions were not allowed. 


\section{Statistical analysis}

Progression-free survival (PFS) was defined as the time from the initiation of pembrolizumab until the date of documented disease progression or death from any cause, whichever occurred first. OS was defined as the time from the initiation of pembrolizumab to death from any cause. Survival probabilities were estimated using the Kaplan-Meier method and compared using the log-rank test. The safety analysis included all patients who visited the clinic at least once after the initiation of treatment. Toxicity was evaluated based on the National Cancer Institute Common Terminology Criteria for Adverse Events ver. 4.03. A p-value of $<0.05$ was considered significant. SPSS ver. 23.0 (IBM Corp., Armonk, NY) was used for statistical analyses.

\section{Ethical statement}

The study was approved by the Institutional Review Board of Asan Medical Center (2018-0257). All patients provided a written informed consent, and the study was conducted in accordance with the Declaration of Helsinki.

\section{Results}

\section{Patient characteristics}

Between May 2018 and February 2019, 40 patients were enrolled in this study. Baseline patient characteristics are summarized in Table 1. The median age was 61 years (range, 41 to 76 years), and $23(57.5 \%)$ patients were men. iCCA was the most common type $(n=20,50 \%)$, followed by GBCA $(\mathrm{n}=12,30 \%)$, and eCCA $(\mathrm{n}=8,20 \%)$. Lymph nodes $(\mathrm{n}=30$, $75 \%)$ and liver $(\mathrm{n}=23,57.5 \%)$ were the most frequent metastatic sites. At the time of pembrolizumab administration, most patients had metastatic disease $(n=38,95 \%)$. More than half of the patients had an Eastern Cooperative Oncology Group performance status of $0-1(n=21,52.5 \%)$.

Pembrolizumab was administered as second-, third-, and fourth-line therapy or more in $19(47.5 \%), 16(40 \%)$, and five $(12.5 \%)$ patients, respectively. All patients received GemCis as first-line systemic therapy; the median time to progression of the first-line GemCis was 5.9 months (95\% confidence interval [CI], 4.1 to 7.8$)$.

\section{Treatment and clinical outcomes}

Median three cycles (range, 1 to 12 ) of pembrolizumab were administered, and disease progression was the most
Table 1. Baseline patient characteristics

\begin{tabular}{|c|c|}
\hline Characteristic & No. $(\%)(n=40)$ \\
\hline Age, median (range, yr) & $61(41-76)$ \\
\hline$<65$ & $26(65.0)$ \\
\hline$\geq 65$ & $14(35.0)$ \\
\hline \multicolumn{2}{|l|}{ Sex } \\
\hline Male & $23(57.5)$ \\
\hline Female & $17(42.5)$ \\
\hline \multicolumn{2}{|l|}{ Primary tumor location } \\
\hline Intrahepatic & $20(50.0)$ \\
\hline Extrahepatic & $8(20.0)$ \\
\hline Gallbladder & $12(30.0)$ \\
\hline \multicolumn{2}{|l|}{ Disease setting at presentation } \\
\hline Metastatic & $38(95.0)$ \\
\hline Locally advanced unresectable & $2(5.0)$ \\
\hline \multicolumn{2}{|l|}{ Histology } \\
\hline Adenocarcinoma & $34(85.0)$ \\
\hline Adenosquamous carcinoma & $3(7.5)$ \\
\hline Others & $3(7.5)$ \\
\hline \multicolumn{2}{|l|}{ ECOG performance status } \\
\hline 1 & $21(52.5)$ \\
\hline$\geq 2$ & $19(47.5)$ \\
\hline \multicolumn{2}{|l|}{ Site of metastasis } \\
\hline Lymph node & $30(75.0)$ \\
\hline Liver & $23(57.5)$ \\
\hline Peritoneum & $17(42.5)$ \\
\hline Bone & $8(20.0)$ \\
\hline Lung & $6(15.0)$ \\
\hline \multicolumn{2}{|l|}{ Differentiation } \\
\hline Well differentiated & $2(5.0)$ \\
\hline Moderately differentiated & $19(47.5)$ \\
\hline Poorly differentiated & $9(22.5)$ \\
\hline Unknown & $10(25.0)$ \\
\hline \multicolumn{2}{|l|}{ MMR status ( $\mathrm{n}=33$ ) } \\
\hline Proficient & $33(100)$ \\
\hline Deficient & 0 \\
\hline Prior curative surgery & $16(40.0)$ \\
\hline \multicolumn{2}{|l|}{ Prior systemic therapies } \\
\hline 1 & $19(47.5)$ \\
\hline 2 & $16(40.0)$ \\
\hline$\geq 3$ & $5(12.5)$ \\
\hline
\end{tabular}

ECOG, Eastern Cooperative Oncology Group; MMR, mismatch repair.

common cause of treatment discontinuation $(\mathrm{n}=31,77.5 \%)$. The waterfall plot for the maximal change of the target lesions among patients with measurable disease $(n=38,95 \%)$ is presented in Fig. 1. Per RECIST v1.1, 39 (97.5\%) patients were assessable for response. None of the patients exhibited complete response, while four achieved partial response 


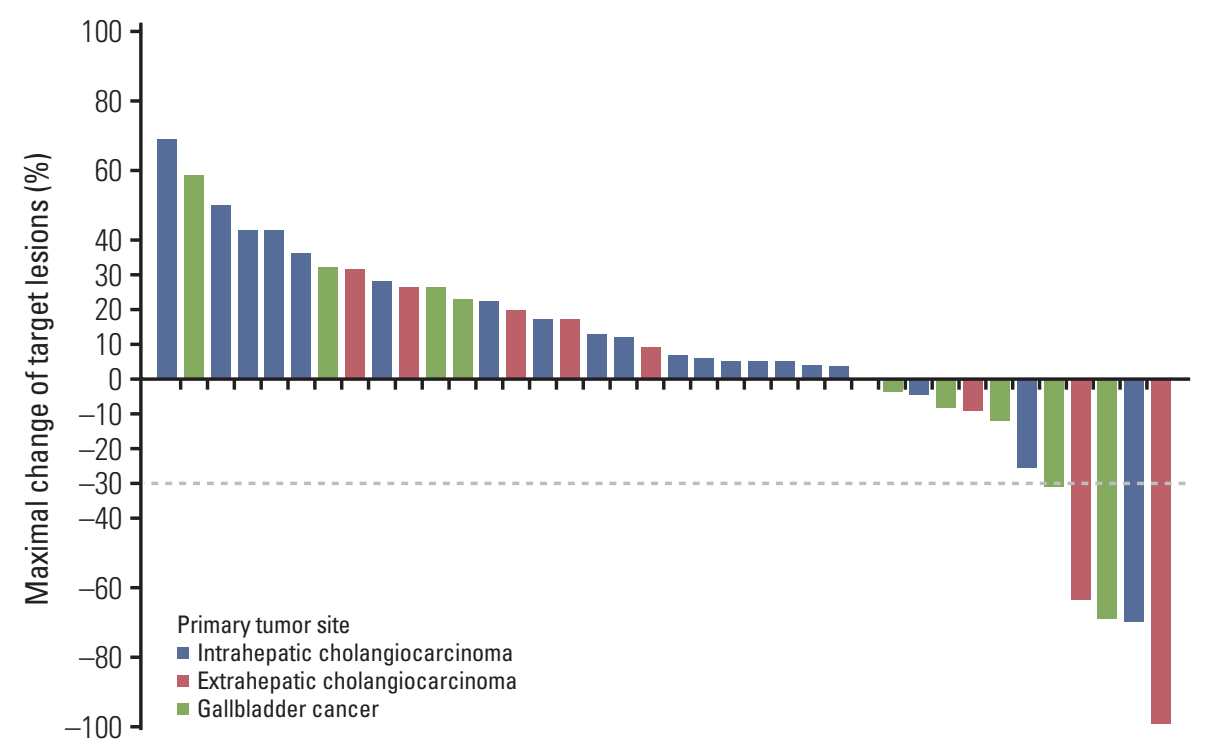

Fig. 1. Waterfall plots of the changes in the size of target lesions.

Table 2. Efficacy outcomes of pembrolizumab according to RECIST v1.1 and imRECIST

$\begin{array}{lcc}\text { Variable } & \text { Per RECIST v1.1 (n=40) } & \text { Per imRECIST (n=40) } \\ \text { Objective response } & & 1(2.5) \\ \text { CR } & 0 & 4(10.0) \\ \text { PR } & 4(10.0) & 18(45.0) \\ \text { SD } & 15(37.5) & 16(40.0) \\ \text { PD } & 20(50.0)^{\mathrm{a})} & 1(2.5) \\ \text { Not evaluable } & 1(2.5) & 12.5 \\ \text { ORR (CR+PR) (\%) } & 10.0 & 1 / 20(5.0) \\ \text { ORR by primary tumor site } & 1 / 20(5.0) & 2 / 8(25.0) \\ \text { Intrahepatic cholangiocarcinoma } & 1 / 8(12.5) & 2 / 12(16.7) \\ \text { Extrahepatic cholangiocarcinoma } & 2 / 12(16.7) & 2.5(1.6-3.4) \\ \text { Gallbladder cancer } & 1.5(0-3.0) & 4.3(3.5-5.1) \\ \text { Progression-free survival (95\% CI, mo) } & 4.3(3.5-5.1) & - \\ \text { Overall survival (95\% CI, mo) } & & \end{array}$

Values are presented as number (\%) or median (95\% CI) unless otherwise indicated. RECIST, Response Evaluation Criteria in Solid Tumors; imRECIST, immune-modified RECIST; CR, complete response; PR, partial response; SD, stable disease; $\mathrm{PD}$, progressive disease; $\mathrm{ORR}$, objective response rate; $\mathrm{CI}$, confidence interval. ${ }^{\text {a) }}$ Among patients with $\mathrm{PD}$, one achieved $\mathrm{CR}$ subsequently with the use of pembrolizumab beyond PD.

(PR), with an objective response rate (ORR) of $10 \%$ (Table 2). Among patients with PD as best response, one $(1 / 20,5 \%)$ with eCCA as primary tumor achieved complete response (CR) subsequently (i.e., pseudoprogression) with the use of pembrolizumab beyond initial PD (Fig. 2). According to imRECIST, ORR was $12.5 \%$ ( 5 of 40 ). The ORR per imRECIST according to primary tumor site were 5\% (1 of 20 patients), $25 \%$ ( 2 of 8 patients), and $16.7 \%$ ( 2 of 12 patients) in patients with iCCA, eCCA, and GBCA, respectively. In patients with CR or PR per imRECIST, the median time to response was 2.1 months (95\% CI, 0.4 to 3.9), and the median duration of response (DOR) was 6.3 months (95\% CI, not available). At the time of analysis, three of five patients who achieved CR or PR, including a patient with pseudoprogression, continued receiving pembrolizumab (5.1+ to $9.6+$ months).

With a median follow-up of 9.6 months ( $95 \%$ CI, 4.5 to 14.6$)$ 


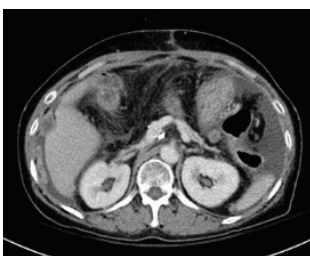

Baseline

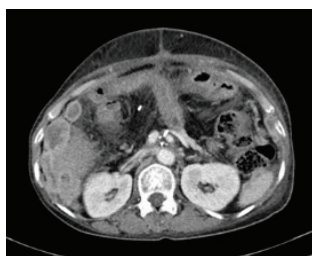

Cycle \#2: PD

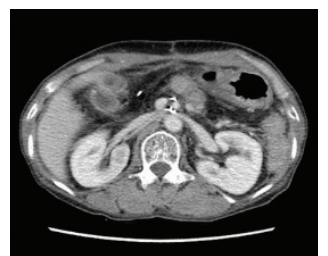

Cycle \#5: CR

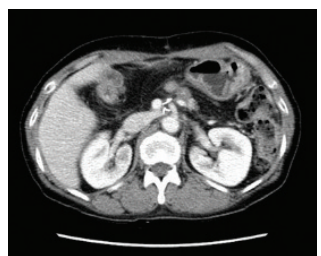

Cycle \#9: CR

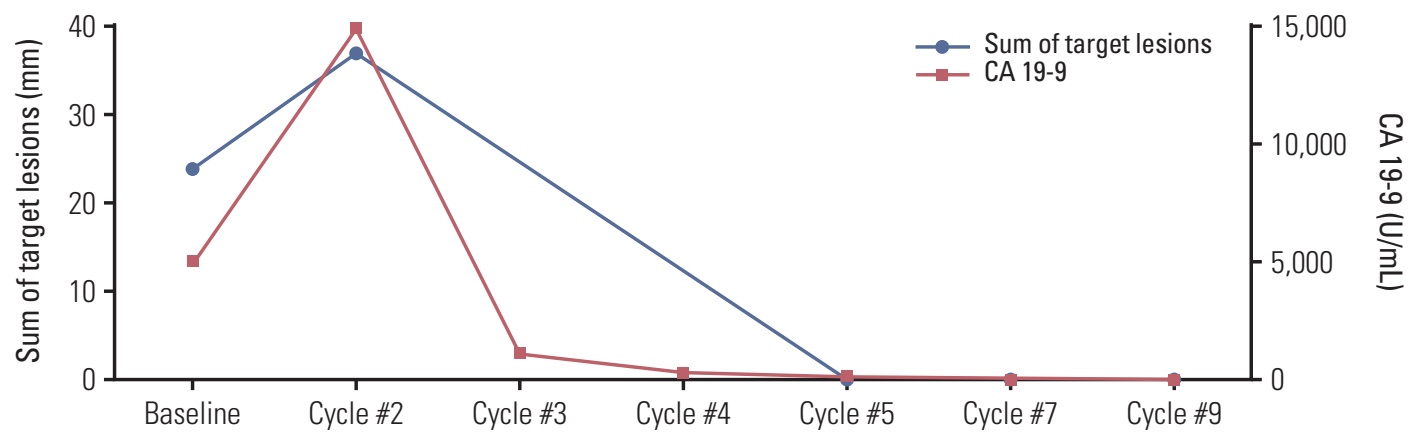

Fig. 2. Flow charts of a patient with pseudoprogression treated with pembrolizumab. Sum of target lesions and serum cancer antigen 19-9 (CA 19-9) levels are depicted with the computed tomography scan images. PD, progressive disease; CR, complete response.

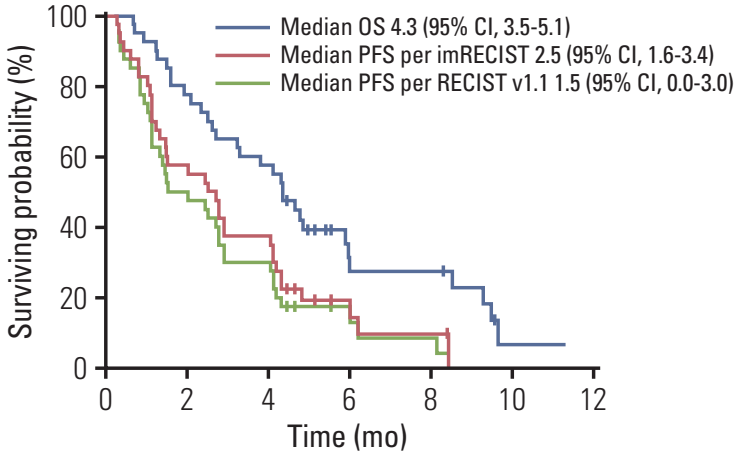

Fig. 3. Progression-free survival (according to Response Evaluation Criteria in Solid Tumor [RECIST] v1.1 and immune-modified RECIST [imRECIST]) and overall survival. OS, overall survival; CI, confidence interval; PFS, progression-free survival.

in surviving patients, the median PFS was 1.5 months (95\% CI, 0.0 to 3.0) per RECIST v1.1 and 2.5 months (95\% CI, 1.6 to 3.4 ) per imRECIST (Table 2, Fig. 3). The median OS was 4.3 months (95\% CI, 3.5 to 5.1). The 6-month PFS rates per RECIST v1.1, and imRECIST were $13.1 \%$ (95\% CI, 1.5 to 24.7 ) and $14.5 \%$ (95\% CI, 2.0 to 27.0 ), respectively, and 6-month OS rates were and $27.5 \%$ (95\% CI, 12.0 to 43.0 ).

There were significant differences in PFS and OS according to the objective response per imRECIST $(p<0.001$ and $\mathrm{p}=0.001$, respectively) (Fig. 4). According to imRECIST, the median PFS and OS were 8.4 months (95\% CI, not available) and 9.5 months (95\% CI, not available), respectively, in patients with CR or PR; 2.9 months (95\% CI, 1.1 to 4.7) and 4.6 months (95\% CI, 3.7 to 5.5), respectively, in those with stable disease (SD); and 1.0 months (95\% CI, 0.5 to 1.5$)$ and 2.1 months (95\% CI, 1.2 to 2.9 ), respectively, in those with PD. In patients with $\mathrm{CR}$ or $\mathrm{PR}, \mathrm{SD}$, and $\mathrm{PD}$, the 6-month $\mathrm{PFS}$ rates were $80 \%$ (95\% CI, 44.9 to 100), $11.1 \%$ (95\% CI, 0.0 to 25.6), and $0 \%$ (95\% CI, 0.0 to 0.0 ), respectively, while the 6-month OS rates were $100 \%$ (95\% CI, not available), $32.4 \%$ (95\% CI, 10.3 to 54.5 ), and $0 \%$ (95\% CI, 0.0 to 0.0 ), respectively.

PFS per imRECIST and OS did not differ according to histologic type (adenocarcinoma vs. others), primary tumor site (iCCA vs. eCCA vs. GBCA), cancer antigen 19-9 level (elevated vs. normal), and number of prior systemic chemotherapy (1 vs. $\geq 2)(p>0.05$ for all).

\section{Correlative biomarker analysis}

Although this study included patients who had tumor PDL1 expression $\geq 1 \%$ by local pathologists, central pathology review revealed that nine $(22.5 \%)$ and two $(5.0 \%)$ patients had a TPS and a CPS of $<1$, respectively. The TPS and CPS of all patients were obtained, the median TPS and CPS were 4.5 (range, 0 to 100) and 20 (range, 0 to 100), respectively. Considering there was a patient with pseudoprogression and this may underestimate the efficacy of pembrolizumab when ORR and PFS were graded by RECIST v1.1, the imRECISTbased efficacy outcomes were used in the correlative bio- 
A
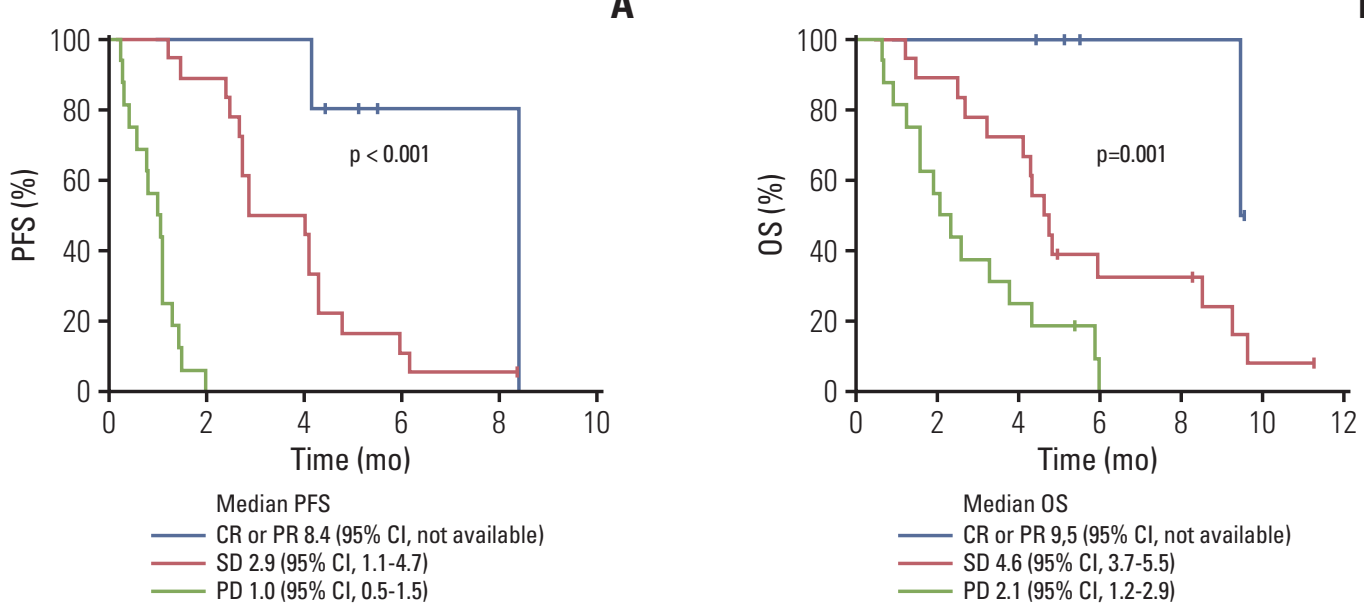

Fig. 4. Progression-free survival (PFS) (A) and overall survival (OS) (B) according to the objective response graded based on immune-modified Response Evaluation Criteria in Solid Tumor. CR, complete response; PR, partial response; SD, stable disease; $\mathrm{PD}$, progressive disease; $\mathrm{CI}$, confidence interval.

Table 3. Correlative analysis between tumor proportion score, combined positive score, and tumor response according to imRECIST

\begin{tabular}{|c|c|c|c|}
\hline Variable & CR or $P^{a)}$ & SD or PD & p-value \\
\hline \multicolumn{4}{|l|}{ TPS } \\
\hline$\geq 1(\mathrm{n}=31)$ & $4(12.9)$ & $27(87.1)$ & $>0.99$ \\
\hline$<1(\mathrm{n}=8)$ & $1(12.5)$ & 7 (87.5) & \\
\hline \multicolumn{4}{|l|}{ TPS } \\
\hline$\geq 20(\mathrm{n}=12)$ & $3(25.0)$ & $9(75.0)$ & 0.159 \\
\hline$<20(\mathrm{n}=27)$ & $2(7.4)$ & 25 (92.6) & \\
\hline \multicolumn{4}{|l|}{ TPS } \\
\hline$\geq 50(\mathrm{n}=8)$ & $3(37.5)$ & $5(62.5)$ & 0.049 \\
\hline$<50(\mathrm{n}=31)$ & $2(6.5)$ & $29(93.5)$ & \\
\hline \multicolumn{4}{|l|}{ CPS } \\
\hline$\geq 1(\mathrm{n}=37)$ & $4(10.8)$ & $33(89.2)$ & 0.243 \\
\hline$<1(\mathrm{n}=2)$ & $1(50.0)$ & $1(50.0)$ & \\
\hline \multicolumn{4}{|l|}{ CPS } \\
\hline$\geq 20(n=21)$ & $3(14.3)$ & $18(85.7)$ & $>0.99$ \\
\hline$<20(\mathrm{n}=18)$ & $2(11.1)$ & $16(88.9)$ & \\
\hline \multicolumn{4}{|l|}{ CPS } \\
\hline$\geq 50(\mathrm{n}=13)$ & $3(23.1)$ & $10(76.9)$ & 0.310 \\
\hline$<50(\mathrm{n}=26)$ & $2(7.7)$ & $24(92.3)$ & \\
\hline
\end{tabular}

Values are presented as number (\%). imRECIST, immune-modified Response Evaluation Criteria in Solid Tumors; CR, complete response; PR, partial response; SD, stable disease; PD, progressive disease; TPS, tumor proportion score; CPS, combined positive score. ${ }^{\mathrm{a}}$ Include a patient who showed $\mathrm{CR}$ after initial $\mathrm{PD}$ with the use of pembrolizumab beyond PD.

marker analysis. In patients with TPS $\geq 1$, the median PFS per imRECIST and OS were 2.4 months (95\% CI, 1.0 to 3.8) and 4.3 months ( $95 \%$ CI, 3.2 to 5.5), respectively. According to imRECIST, ORR was significantly higher among patients with TPS $\geq 50 \%$ ( $37.5 \%$ [ 3 of 8 patients] vs. $6.5 \%$ in TPS $<50 \%$ [ 2 of 31 patients]; $\mathrm{p}=0.049$ ) (Table 3 ) and the median PFS was significantly longer in patients with TPS $\geq 50 \%$ (2.9 months [ $95 \%$ CI, 0.0 to 6.7] vs. 2.4 months [ $95 \%$ CI, 0.8 to 4.1 ] in TPS 
A
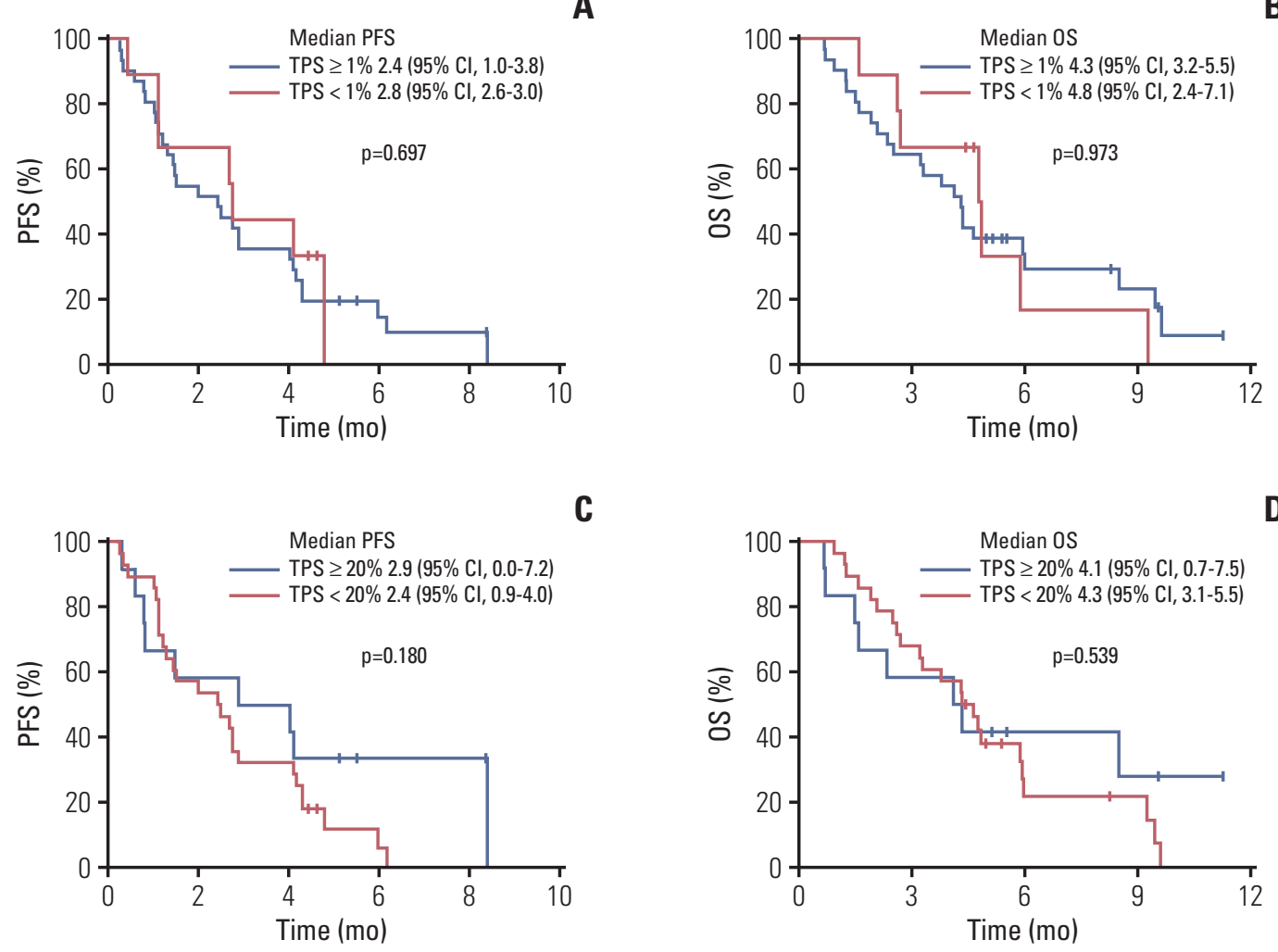

$\mathbf{E}$
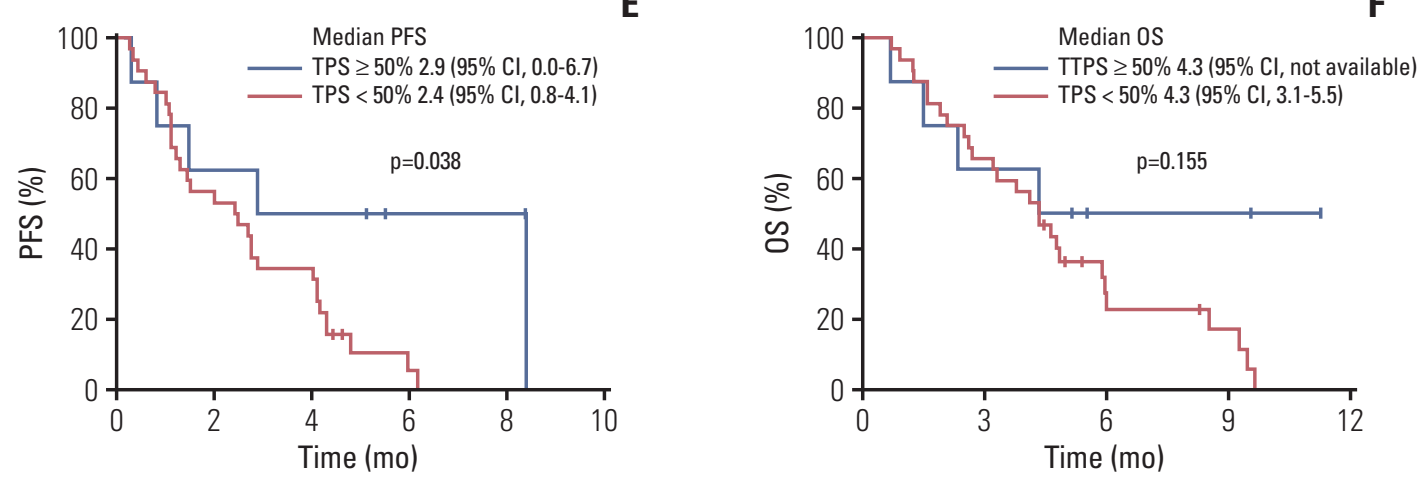

Fig. 5. Progression-free survival (PFS) per immune-modified Response Evaluation Criteria in Solid Tumor and overall survival (OS) according to the tumor proportion score (TPS) of programmed death ligand-1. Cut-off at 1\% (A, B), 20\% (C, D), and $50 \%$ (E, F). CI, confidence interval.

$<50 \% ; \mathrm{p}=0.038$ ) (Fig. 5). OS did not differ according to the cut-off values $(1,20$, and 50$)$ of TPS and CPS ( $p>0.05$ for all).

The MMR status of 33 patients $(82.5 \%)$ were assessable, while seven patients lacked archival tissues for further immunohistochemical staining of MMR proteins. Among these patients, none showed MMR deficiency; therefore, correlative analysis between MMR status and the efficacy of pembrolizumab could not be performed.

\section{Adverse events}

Data on the safety of pembrolizumab were available in 39 patients. While none of the patients experienced grade 3-5 adverse events (AEs), 8 (20.5\%) experienced treatmentrelated AEs: fatigue $(n=4,10.3 \%)$ and pruritis $(n=4,10.3 \%)$ were the most frequent AEs. None of the patients experienced immune-related AEs, and no AE-related treatment delays or interruptions were reported. 


\section{Discussion}

In this study, pembrolizumab showed a modest anti-tumor activity and manageable AEs in heavily pretreated patients with advanced BTC. None of the patients treated with pembrolizumab developed new AEs, and response was durable for patients who achieved objective response.

In this prospective cohort study including 40 BTC patients with $77.5 \%$ of PD-L1-positive $(\geq 1)$ by central pathology review, ORR was $10 \%$ per RECIST v1.1 and $12.5 \%$ per imRECIST. The median PFS was 1.5 months per RECIST v1.1 and 2.5 months per imRECIST, and OS was 4.3 months. Considering that our study patients were heavily pretreated as more than half of the patients $(52.5 \%)$ received pembrolizumab as third-line chemotherapy or greater, our efficacy results seem to be comparable with the clinical outcomes in other prospective clinical trials using pembrolizumab for pretreated patients with advanced BTC; the KEYNOTE-028 study of 24 patients with PD-L1-positive advanced BTC showed an ORR of $13 \%$ per RECIST v1.1 and median PFS and OS of 1.8 months and 5.7 months, respectively [22]. The KEYNOTE158 study of 104 patients with $58.7 \%$ PD-L1-positive rates showed an ORR of $5.8 \%$ per RECIST v1.1 and median PFS and $O S$ of 2.0 months and 7.4 months, respectively [22]. In a recent Japanese phase 1 study of another anti-PD-1 inhibitor nivolumab, which included 30 patients with PD-L1-positive rates of $18 \%$, also showed consistent efficacy outcomes, with ORR of $3.3 \%$ per RECIST v1.1 and median PFS and OS of 1.4 months and 5.2 months, respectively [26].

The discrepancies in efficacy outcomes among the studies that investigated the efficacy of anti-PD-1 antibody in advanced BTC may be mainly attributable to the differences in baseline patient characteristics considering that BTC is an extremely heterogeneous disease. Although the role of PDL1 expression as a predictive biomarker in advanced BTC patients treated with PD-1 inhibitor remains unclear, the discrepancies in the proportion of patients with PD-L1 among the studies may have an impact on the efficacy outcomes. The ORRs per RECIST v1.1 were higher in KEYNOTE-028 and our current studies of pembrolizumab (13\% and 10\%, respectively). The PD-L1-positive rates of patients included in these studies were $100 \%$ and $77.5 \%$, respectively, while those in KEYNOTE-158 study of pembrolizumab (ORR 5.8\%) and Japanese phase 1 study of nivolumab (ORR 3.3\%) were $58.7 \%$ and $17.8 \%$, respectively. ORR was higher in PD-L1positive patients (6.6\% vs. $2.9 \%$ ) in the KEYNOTE-158 study [22]. In the Japanese phase 1 study of nivolumab, PD-L1-positive patients had longer median PFS (2.8 months vs. 1.4 months) and OS (11.6 months vs. 5.2 months) than PD-L1negative patients [26]. Our analysis also showed an increase in ORR and PFS per imRECIST in higher cut-offs of TPS.
However, these findings were based on the preliminary studies with relatively small sample size. Hence, further analyses using large randomised clinical trials are needed to determine the implication of tumor PD-L1 status in BTC patients treated with anti-PD-1 inhibitors.

Cancer patients with MMR deficiency are associated with somatic hypermutation and neoepitope formation leading to microsatellite instability (MSI) [27], and PD-1 blockades showed $40 \%-50 \%$ of ORR in patients with solid cancers harboring deficient MMR after progression on conventional therapies [16,17]. As the frequency of MMR deficiency or MSI-high in BTC was reported to be $2 \%-10 \%$ [18-20], these can be a relevant biomarker for the use of PD-1 blockades in BTC patients. In the current study, MMR status was assessed by immunohistochemistry [28]. However, none of the patients showed MMR deficiency in our study, and this result is in line with those of KEYNOTE-028 and KEYNOTE-158 studies, which reported that only $1(0.8 \%)$ of 128 patients had MSI-H [22].

In previous studies that adopted pembrolizumab as a salvage therapy for pretreated BTC patients including ours, pembrolizumab showed a modest efficacy with ORR of $6 \%$ $13 \%$, median PFS of 1.5-2.0 months, and median OS of 4.37.3 months. Fluoropyrimidine-based chemotherapy showed median PFS of 1.9 months and OS of 6.5 months in a previous retrospective analysis [29], while mFOLFOX showed median PFS and OS of 4.0 and 6.2 months, respectively; in a recent randomized phase $3 \mathrm{ABC}-06$ trial after progression on firstline GemCis [5], current evidences with pembrolizumab are not compelling for its use as salvage therapy in overall BTC patients. However, pembrolizumab may have clinical relevance in the management of refractory BTC patients in terms of long DOR (median DOR of 6.3 months in our study, and 2-year DOR of $67 \%$ and 50\% in the KEYNOTE-028 and KEYNOTE-158 studies, respectively) and better safety profile compared with mFOLFOX (grades 3-4 AEs: 14\%-17\% in KEYNOTE-028 and KEYNOTE-158 studies vs. 59\% in the ABC-06 trial). Extensive biomarker analysis to define the patient population who would benefit most should be performed in the future.

There are several caveats in our study. Although our study prospectively evaluated the safety and efficacy of pembrolizumab, this was conducted at a single center. Our patient population is heterogeneous, and many of them previously received a variety of treatments. Additionally, the sample size was not enough to conduct a statistically robust analysis for a correlative study.

In conclusion, pembrolizumab showed a modest efficacy in heavily pretreated PD-L1-positive BTC patients. Pembrolizumab was well tolerated, and no new AEs occurred in these fragile patient population. Future studies for improving anti-PD-1 inhibitors and finding biomarkers in BTC pati- 
ents are warranted.

\section{Conflicts of Interest}

Conflict of interest relevant to this article was not reported.

\section{Acknowledgments}

This study was funded in part by grants from the Bio and Medical Technology Development Program of the National Research Foundation (NRF) of Korea, funded by the Ministry of Science, Communications Technology, and Future Planning of the Korean government (NRF-2016M3A9E8941331), and Asan Institute for Life Sciences at Asan Medical Center in Seoul, Korea (2018-0634).

\section{References}

1. Razumilava N, Gores GJ. Cholangiocarcinoma. Lancet. 2014; 383:2168-79.

2. Siegel RL, Miller KD, Jemal A. Cancer statistics, 2018. CA Cancer J Clin. 2018;68:7-30.

3. Patel T. Increasing incidence and mortality of primary intrahepatic cholangiocarcinoma in the United States. Hepatology. 2001;33:1353-7.

4. Edge SB, Compton CC. The American Joint Committee on Cancer: the 7th edition of the AJCC cancer staging manual and the future of TNM. Ann Surg Oncol. 2010;17:1471-4.

5. Lamarca A, Palmer DH, Wasan HS, Ross PJ, Ma YT, Arora A, et al. ABC-06 I A randomised phase III, multi-centre, openlabel study of active symptom control (ASC) alone or ASC with oxaliplatin / 5-FU chemotherapy (ASC+mFOLFOX) for patients (pts) with locally advanced / metastatic biliary tract cancers $(\mathrm{ABC})$ previously-treated with cisplatin/gemcitabine (CisGem) chemotherapy. J Clinl Oncol. 2019;37(15 Suppl): 4003.

6. Valle J, Wasan H, Palmer DH, Cunningham D, Anthoney A, Maraveyas A, et al. Cisplatin plus gemcitabine versus gemcitabine for biliary tract cancer. N Engl J Med. 2010;362:127381.

7. Valle JW, Borbath I, Khan SA, Huguet F, Gruenberger T, Arnold D, et al. Biliary cancer: ESMO Clinical Practice Guidelines for diagnosis, treatment and follow-up. Ann Oncol. 2016;27(Suppl 5):v28-37.

8. Hyung J, Kim B, Yoo C, Kim KP, Jeong JH, Chang HM, et al. Clinical benefit of maintenance therapy for advanced biliary tract cancer patients showing no progression after first-line gemcitabine plus cisplatin. Cancer Res Treat. 2019;51:901-9.

9. Kim BJ, Hyung J, Yoo C, Kim KP, Park SJ, Lee SS, et al. Prognostic factors in patients with advanced biliary tract cancer treated with first-line gemcitabine plus cisplatin: retrospective analysis of 740 patients. Cancer Chemother Pharmacol. 2017; 80:209-15.

10. Eggermont AM, Blank CU, Mandala M, Long GV, Atkinson $\mathrm{V}$, Dalle $\mathrm{S}$, et al. Adjuvant pembrolizumab versus placebo in resected stage III melanoma. N Engl J Med. 2018;378:1789-801.

11. Bellmunt J, de Wit R, Vaughn DJ, Fradet Y, Lee JL, Fong L, et al. Pembrolizumab as second-line therapy for advanced urothelial carcinoma. N Engl J Med. 2017;376:1015-26.
12. Muro K, Chung HC, Shankaran V, Geva R, Catenacci D, Gupta $\mathrm{S}$, et al. Pembrolizumab for patients with PD-L1-positive advanced gastric cancer (KEYNOTE-012): a multicentre, openlabel, phase 1b trial. Lancet Oncol. 2016;17:717-26.

13. Garon EB, Rizvi NA, Hui R, Leighl N, Balmanoukian AS, Eder JP, et al. Pembrolizumab for the treatment of non-small-cell lung cancer. N Engl J Med. 2015;372:2018-28.

14. Herbst RS, Soria JC, Kowanetz M, Fine GD, Hamid O, Gordon MS, et al. Predictive correlates of response to the anti-PD-L1 antibody MPDL3280A in cancer patients. Nature. 2014;515: 563-7.

15. Topalian SL, Hodi FS, Brahmer JR, Gettinger SN, Smith DC, McDermott DF, et al. Safety, activity, and immune correlates of anti-PD-1 antibody in cancer. N Engl J Med. 2012;366:244354.

16. Le DT, Durham JN, Smith KN, Wang H, Bartlett BR, Aulakh LK, et al. Mismatch repair deficiency predicts response of solid tumors to PD-1 blockade. Science. 2017;357:409-13.

17. Le DT, Uram JN, Wang H, Bartlett BR, Kemberling H, Eyring $\mathrm{AD}$, et al. PD-1 blockade in tumors with mismatch-repair deficiency. N Engl J Med. 2015;372:2509-20.

18. Silva VW, Askan G, Daniel TD, Lowery M, Klimstra DS, Abou-Alfa GK, et al. Biliary carcinomas: pathology and the role of DNA mismatch repair deficiency. Chin Clin Oncol. 2016;5:62.

19. Salem ME, Puccini A, Grothey A, Raghavan D, Goldberg RM, Xiu J, et al. Landscape of tumor mutation load, mismatch repair deficiency, and PD-L1 expression in a large patient cohort of gastrointestinal cancers. Mol Cancer Res. 2018;16: 805-12.

20. Goeppert B, Roessler S, Renner M, Singer S, Mehrabi A, Vogel $M N$, et al. Mismatch repair deficiency is a rare but putative therapeutically relevant finding in non-liver fluke associated cholangiocarcinoma. Br J Cancer. 2019;120:109-14.

21. Fontugne J, Augustin J, Pujals A, Compagnon P, Rousseau B, Luciani A, et al. PD-L1 expression in perihilar and intrahepatic cholangiocarcinoma. Oncotarget. 2017;8:24644-51.

22. Bang YJ, Ueno M, Malka D, Chung HC, Nagrial A, Kelley RK, et al. Pembrolizumab (pembro) for advanced biliary adenocarcinoma: results from the KEYNOTE-028 (KN028) and KEYNOTE-158 (KN158) basket studies. J Clin Oncol. 2019; 
37(15 Suppl):4079.

23. Scheel AH, Dietel M, Heukamp LC, Johrens K, Kirchner T, Reu S, et al. Harmonized PD-L1 immunohistochemistry for pulmonary squamous-cell and adenocarcinomas. Mod Pathol. 2016;29:1165-72.

24. Dolled-Filhart M, Roach C, Toland G, Stanforth D, Jansson M, Lubiniecki GM, et al. Development of a companion diagnostic for pembrolizumab in non-small cell lung cancer using immunohistochemistry for programmed death ligand-1. Arch Pathol Lab Med. 2016;140:1243-9.

25. Kulangara K, Zhang N, Corigliano E, Guerrero L, Waldroup $\mathrm{S}$, Jaiswal D, et al. Clinical utility of the combined positive score for programmed death ligand-1 expression and the approval of pembrolizumab for treatment of gastric cancer. Arch Pathol Lab Med. 2019;143:330-7.

26. Ueno M, Ikeda M, Morizane C, Kobayashi S, Ohno I, Kondo $S$, et al. Nivolumab alone or in combination with cisplatin plus gemcitabine in Japanese patients with unresectable or recur- rent biliary tract cancer: a non-randomised, multicentre, openlabel, phase 1 study. Lancet Gastroenterol Hepatol. 2019;4: 611-21.

27. Dudley JC, Lin MT, Le DT, Eshleman JR. Microsatellite instability as a biomarker for PD-1 blockade. Clin Cancer Res. 2016; 22:813-20.

28. Luchini C, Bibeau F, Ligtenberg MJ, Singh N, Nottegar A, Bosse $\mathrm{T}$, et al. ESMO recommendations on microsatellite instability testing for immunotherapy in cancer, and its relationship with PD-1/PD-L1 expression and tumour mutational burden: a systematic review-based approach. Ann Oncol. 2019;30:1232-43.

29. Kim BJ, Yoo C, Kim KP, Hyung J, Park SJ, Ryoo BY, et al. Efficacy of fluoropyrimidine-based chemotherapy in patients with advanced biliary tract cancer after failure of gemcitabine plus cisplatin: retrospective analysis of 321 patients. Br J Cancer. 2017;116:561-7. 